\title{
Globalização, cultura e território: o Brasil no novo milênio
}

Globalization, culture and territory: Brazil in the new millennium

Globalización, cultura y territorio: Brasil en el nuevo milenio

Mondialisation, culture et territoire: le Brésil au nouveau millénaire

\section{Hindenburgo Francisco Pires}

\section{OpenEdition}

Journals

\section{Edição electrónica}

URL: http://journals.openedition.org/espacoeconomia/3031

DOI: 10.4000/espacoeconomia.3031

ISSN: 2317-7837

\section{Editora}

Núcleo de Pesquisa Espaço \& Economia

Refêrencia eletrónica

Hindenburgo Francisco Pires, « Globalização, cultura e território: o Brasil no novo milênio », Espaço e

Economia [Online], 11 | 2017, posto online no dia 05 abril 2018, consultado o 02 maio 2019. URL :

http://journals.openedition.org/espacoeconomia/3031; DOI : 10.4000/espacoeconomia.3031

Este documento foi criado de forma automática no dia 2 Maio 2019

(C) NUPEE 


\title{
Globalização, cultura e território: o Brasil no novo milênio
}

\author{
Globalization, culture and territory: Brazil in the new millennium \\ Globalización, cultura y territorio: Brasil en el nuevo milenio \\ Mondialisation, culture et territoire: le Brésil au nouveau millénaire \\ Hindenburgo Francisco Pires
}

\section{Introdução}

1 Em 2001, quando escrevi mais densamente um artigo sobre as origens do atual processo de globalização "Ethos e mitos do pensamento único globaltotalitário"2, admiti, naquela época, que não havia consenso sobre a origem da globalização, o que havia eram dificuldades de uma formulação científica e embates entre vertentes políticas e discursos identificados com ideologias e concepções sociais de mundo.

2 A grande maioria dessas vertentes políticas visava, ideológica ou filosoficamente, oferecer um conjunto de alternativas para enfrentar/resistir ou se adaptar/ajustar à globalização e aos desígnios da competitividade espúria do capitalismo (Figura 1).

Figura 1. Vertentes politicas no capitalismo periférico brasileiro

\section{Resistir}

Defender o Estado Social

Garantir parcialmente a reprodução social

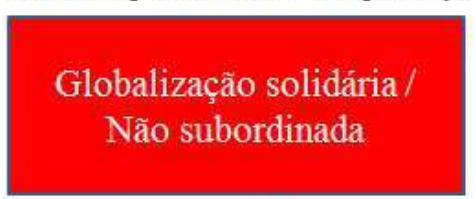

Vertente desenvolvimentista

\section{Adaptar-se}

Implementar a ideologia do Estado mínimo Garantir a acumulação global do capital

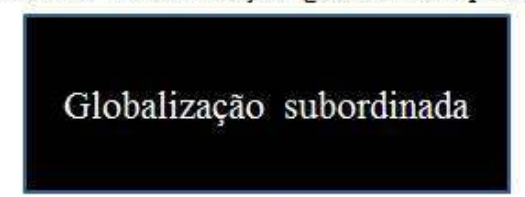

Vertente ultraneoliberalista

ELABORAÇÃO: O AUTOR. 
3 Assim, há vertentes políticas que postulam que é preciso enfrentar a globalização para evitar o aprofundamento da competitividade destrutiva e do crescimento das desigualdades em escala global e também o enfraquecimento da capacidade dos estados em garantir a reprodução social; ou seja, a globalização deve ser conduzida de forma não subordinada e soberana pelos estados nacionais. Na América do Sul, quem apoia esta perspectiva política são setores nacionalistas, intelectuais, sindicatos, entidades da sociedade civil organizada e partidos políticos de centro esquerda, que defendem o controle estratégico pelo estado, de empresas públicas nacionais e a preservação do estado social.

4 Entretanto, há também as vertentes pró-mercado/ultraneoliberais que acreditam que a globalização é um imperativo irreversível do mercado e das empresas globais e que o papel dos governos, neste cenário, é se adaptar ao novo paradigma organizacional das empresas globais ${ }^{3}$, promovendo a constituição de uma escala de fatores favoráveis ao desenvolvimento das vantagens competitivas nacionais.

5 Assim, esta perspectiva defende que o estado deve adotar medidas de ajustamentos "subordinados" aos imperativos da globalização. Em geral, nesta perspectiva política, estão: intelectuais vinculados ao status quo do poder midiático, empresários, representantes de instituições financeiras multilaterais (FMI, Banco Mundial, etc.), grandes grupos financeiros nacionais e internacionais, setores vinculados à mídia internacional e partidos políticos de direita, que defendem a privatização, a liberalização e a internacionalização dos serviços de utilidade pública nos estados nacionais.

6 Neste trabalho defendo, como Milton Santos ${ }^{4}$, a premissa de que é preciso resistir e desenvolver uma globalização solidária e não subordinada, que promova um conjunto de políticas públicas alternativas que possibilitem a formação de uma sociedade com menos desigualdades na distribuição de renda e que também promovam a elevação no padrão de vida dos grupos sociais prejudicados com as reformas atuais das relações de trabalho e perda dos benefícios previdenciários assegurados pela legislação.

\section{Desafios da globalização não subordinada}

7 Durante pelo menos 13 anos (2003-2016), o Brasil vinha experimentando outra forma de globalização não subordinada. No modelo de governança adotado nesse período, o espectro político da governabilidade estatal foi sempre um feixe de tensionamentos, de demandas políticas e um campo de forças de interesses conflitantes.

8 Às vezes, esses tensionamentos podem representar um desafio, quando uma clivagem de forças no poder não pesa na balança contra a estabilidade institucional constituída; ou podem ser um pesadelo, quando esta clivagem de forças põe em risco os projetos políticos erigidos pelo estado social democrático, ou quando também põe em risco a soberania nacional, os projetos e motores econômicos que garantem a empregabilidade, o crescimento, o desenvolvimento econômico e a estabilidade social (Figura 2). 
Figura - 2. Desafios da globalização não subordinada

Brasil: 13 Anos de

Globalização não subordinada - 2003/2016

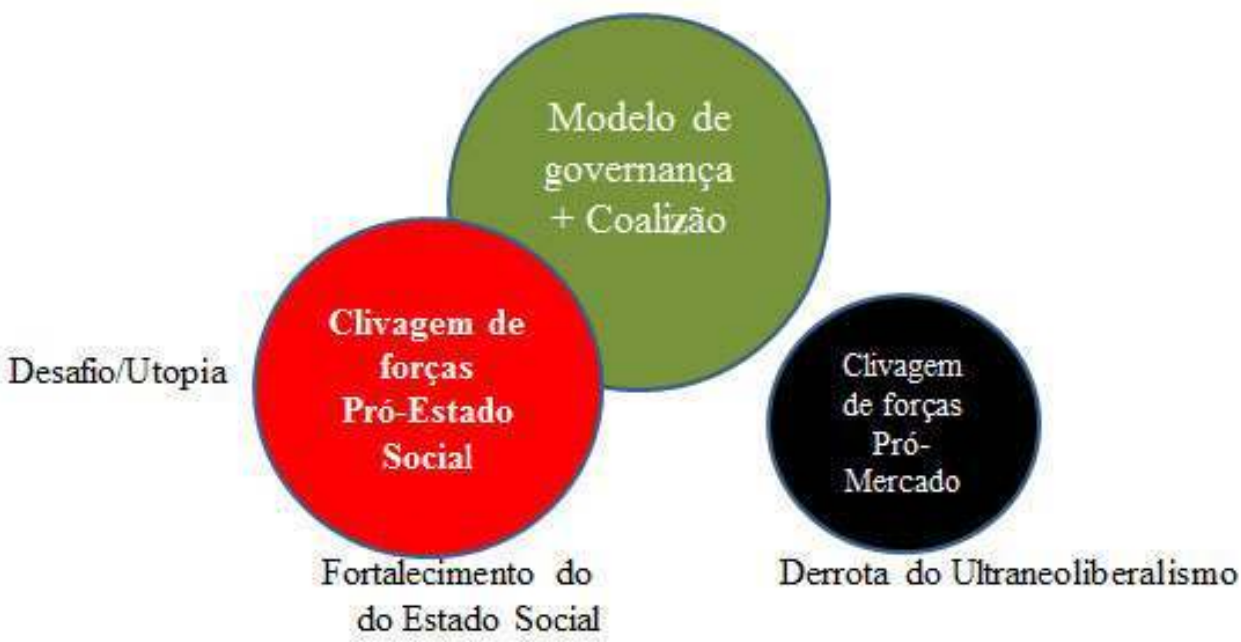

ELABORAÇÃO: O AUTOR.

No contexto político atual do Brasil, a perda de governabilidade foi resultante da destruição dos elementos de coesão política, que mantinham o equilíbrio das forças no giro das negociações políticas do governo no estado, resultando no golpe de estado desfechado em 31 de agosto de 2016, contra a presidente Dilma Rousseff.

Sob a perspectiva da geopolítica, um dos pressupostos centrais do governo da presidente impedida, se assentava na conviç̧ão de que a governabilidade seria o resultado de um processo de coesão gerado a partir de uma coalizão de setores representativos e de diferentes vertentes políticas (de direita e de centro esquerda).

O mais importante nessa posição eram os princípios ou os fundamentos programáticos garantidores da ampliação da zona de influência regional do Brasil, baseados em um viés desenvolvimentista que dava protagonismo aos setores industriais estratégicos da cadeia produtiva nacional (petróleo, gás e construção civil), e mantenedores do estado social, das políticas de reprodução e de proteção social (bolsa família, minha casa minha vida, etc.).

Como os princípios que garantiam esta coalizão passaram a ser questionados pelos setores que davam substância à governabilidade e à gestão pública, esta deixou de ser um desafio, fruto de um permanente diálogo com as forças garantidoras da coalizão, e se transformou literalmente em um pesadelo.

\section{0 pesadelo atual da restauração da globalização subordinada}

A prática política também se transformou em um pesadelo, os vínculos mínimos de confiança desapareceram. Esta transformação suscetibilizou a coesão que garantia o sentido da governabilidade e teve certamente influência geopolítica na redução da zona de influência recente do Brasil no BRICS e no mundo. 
14 No momento atual, a instabilidade gerada pela crise política ameaça e fragiliza a projeção internacional do Brasil. 0 impedimento da presidenta, sem crime de responsabilidade, foi à demonstração cabal de que houve uma ruptura da estabilidade constitucional estabelecida e de que a clivagem de forças que garantia a governabilidade erodiu (Figura 3), e quando não há mais estado de direito prevalece o estado de exceção (AGAMBEN, 2004).

Figura - 3. O pesadelo da globalização subordinada Brasil: Periodo atual - Golpe de Estado Globalização subordinada - 2016/2017

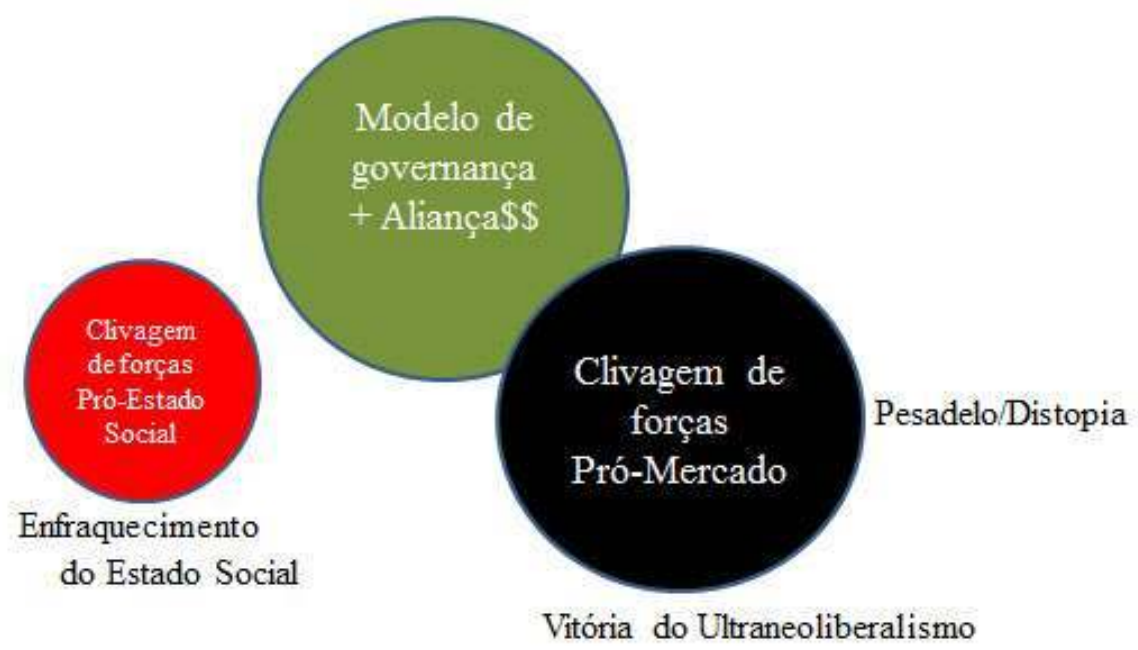

ELABORAÇÃO: O AUTOR.

A perda da governabilidade ocorreu também pelo enfraquecimento do estado de direito, quando um juizado no Paraná utilizou-se de métodos de exceção, baseados na coação dos investigados por práticas de corrupção, para que esses denunciassem, pelo instrumento jurídico da delação "premiada", atividades ilícitas cometidas por setores partidários da coalizão e dirigentes de três importantes multinacionais brasileiras, que respondiam em 2015, por mais de $8 \%$ do faturamento do PIB brasileiro: a primeira, a Petrobras, a $3^{\mathrm{a}}$ maior multinacional brasileira, com 81 mil empregados; a segunda, a Camargo Correa, a $7^{\mathrm{a}}$ maior multinacional brasileira, com 65 mil empregados; a terceira, a Odebrecht, a $8^{\text {a }}$ maior multinacional brasileira, com 181 mil empregados.

O impacto desta perda de governabilidade se refletiu no lucro das multinacionais brasileiras, no produto interno bruto, nos investimentos de longo prazo, na empregabilidade, na dinâmica econômica do consumo e, por conseguinte, no processo de confiança, que mantinha o equilíbrio dos princípios constitucionais que garantia uma coalizão das forças que sustentavam a governabilidade e o respeito aos preceitos constitucionais da democracia no Brasil. 


\section{Impactos da globalização subordinada na educação e na cultura}

17 A educação e a cultura são componentes basilares de expressão e formação social de um povo, defender estes setores com políticas públicas dirigidas ao desenvolvimento social, é fundamental para preservar esses dois importantes valores formadores da identidade de uma nação.

Um mês e dois dias após a decisão do Senado que autorizava a abertura do processo de impeachment, e o impedimento da presidente Dilma Rousseff pelo período de até 180 dias, em 12 de maio de 2016. 0 então vice-presidente Michel Temer, que passou a exercer as funções de presidente da República em exercício, monta uma nova equipe ministerial e começa a fazer valer as promessas contidas no projeto "Uma Ponte para o Futuro" ${ }^{5}$, com propostas de implantação de um novo regime fiscal de austeridade, que tinha como meta a redução do insustentável e generoso regime de bem-estar social, por meio da aprovação de Propostas de Emenda à Constituição (PECs).

Dando continuidade a política de cortes de gastos, o governo golpista, de Michel Temer, ao anunciar a formação de novos ministérios, extinguiu a pasta do Ministério da Cultura (MinC) e a incluiu no Ministério da Educação. O impacto da extinção do MinC foi um retrocesso político difícil de ser dimensionado, a última vez que isso ocorreu, foi no período da ditadura militar, entre o final dos anos 60 e início dos anos 70.

$\mathrm{O}$ antigo MinC possuía, em sua estrutura organizacional, seis importantes secretarias:

21 a) Secretaria da Cidadania e da Diversidade Cultural (SCDC): cujo objetivo era fortalecer o protagonismo cultural da sociedade brasileira;

b) Secretaria do Audiovisual (SAv): concebida para elaborar a política nacional do cinema e do audiovisual;

c)Secretaria de Fomento e Incentivo à Cultura (Sefic): responsável por dar publicidade à alocação, fomento e incentivo à cultura através do Fundo Nacional da Cultura;

d) Secretaria de Articulação e Desenvolvimento Institucional (Sadi): promovia a articulação federativa de programas, projetos e ações culturais executados pela União e pelos estados;

e) Secretaria da Economia da Cultura (SEC): era responsável pelo planejamento, promoção, implementação e coordenação de ações para o desenvolvimento da economia da cultura no País;

f) Secretaria de Infraestrutura Cultural (Seinfra): cujo objetivo era construir, reformar e modernizar equipamentos e bens culturais.

O desmantelamento do sistema nacional de cultura tem implicações políticas na elaboração dos planos e na organização dos conselhos municipais de cultura. Os recursos destinados para a área de cultura e de educação foram drasticamente reduzidos.

Mas, a destruição do MinC não ocorreu sem a resistência da classe artística e dos segmentos vinculados à produção cultural brasileira, uma vez que vários movimentos contrários à extinção do MinC ocuparam, em 18 estados, os prédios destinados ao funcionamento da pasta. 

eliminação também do Ministério das Mulheres, da Igualdade Racial e dos Direitos Humanos, criado para dar visibilidade e garantir o direito de minorias, o empoderamento dos grupos excluídos, e b) a nomeação de apenas homens brancos para os cargos nos 23 ministérios, assumindo um discurso machista com relação as mulheres consideradas "donas do lar", apoiado pela mídia oficial, que deturpava os fatos e os protestos em todo o país, e os índices de rejeição contra o governo.

Pressionado por um forte movimento social nacional de defesa do MinC, o governo federal resolveu recriar novamente por Medida Provisória (MP-728/2016) o Minc. Com a edição dessa MP, o MinC deixou de ser uma secretaria do Ministério da Educação.

31 Em 12 de dezembro de 2016, o governo federal promulgou a PEC $241 / 55^{6}$, que estabeleceu um teto para os gastos públicos, que congela as despesas do Governo Federal por até 20 anos. Em abril de 2017, o governo federal anunciou, segundo o Andes ${ }^{7}$ :

“... um corte de $\mathrm{R} \$ 42,1$ bilhões no orçamento aprovado para o ano de 2017.

o Ministério da Educação (MEC) teve um dos maiores cortes: $\mathrm{R} \$ 4,3$ bilhões, o que representa uma diminuição de $12 \%$ no montante anteriormente definido em $\mathrm{R} \$$ 35,74 bilhões.

o Ministério da Defesa perdeu R\$5,75 bilhões, o Ministério das Cidades teve corte de $\mathrm{R} \$ 4,17$ bilhões, o Ministério dos Transportes, Portos e Aviação Civil de R $\$ 5,13$ bilhões, e o Ministério do Desenvolvimento Social e Agrário perdeu R $\$ 2,25$ bilhões. Houve cortes menores em outras pastas - a única que escapou do ajuste foi a de Saúde."

es cortes orçamentários representaram a consolidação do novo regime fiscal de austeridade e o início do processo de redução dos investimentos da União, nos setores vinculados à educação pública e gratuita, e também, em vários setores instituições $\mathrm{e}$ companhias estatais.

\section{Impactos da globalização subordinada no território}

No atual governo golpista, os 13 anos de vigência do estado social no Brasil estão sendo rapidamente destruídos pela imposição de um projeto neoliberal, fundamentado nos processos de internacionalização e privatização de setores estratégicos da economia (petróleo, gás e construção civil). A perda da governabilidade e a instabilidade jurídica propiciaram à ascensão de setores pró-mercado/ultraneoliberais favoráveis à globalização subordinada.

Os setores ultraneoliberais defendem o discurso de que o estado mínimo é uma necessidade, erigida para permitir a estabilidade da economia e atrair investidores externos globais, mas na verdade, as políticas desenvolvidas por esses setores visam o interesse particular dos empresários e representantes do capital financeiro nacional e internacional.

35 A globalização subordinada ocorre quando se constitui um arco de alianças entre as burguesias nacionais e internacionais, isso não significa que o poder do estado tenha terminado, a modernização conservadora do estado ocorre devido a crescente internacionalização do capital. Essa aliança não prescinde do poder do estado, pelo contrário, o estado burguês (HEIDEMANN, 1983) sofre uma reapropriação institucional, passando a estabelecer juridicamente reformas e políticas favoráveis a reprodução ampliada do capital (JESSOP, 1998, p. 19-20). 

exteriores pretendem conduzir uma política dirigida para extinguir a política externa multilateral, sul-sul, para voltar à subsunção recolonizadora do Brasil, através de uma política externa unilateral que pretende restaurar os postulados da globalização neoliberal fundamentados no Consenso de Washington, e na antiga proeminência dos interesses do norte (EUA - via Banco Mundial - BM, Europa - via Fundo Monetário Internacional - FMI) sobre o sul.

Essa forma de subsunção terá implicações na autonomia do poder do estado brasileiro. A perda de governabilidade poderá trazer implicações preocupantes no projeto de integração econômico e geopolítico do BRICS ${ }^{8}$, pois a política externa multilateral via Novo Banco de Desenvolvimento do BRICS, que dava projeção regional e autonomia ao capitalismo periférico do Brasil no sistema-mundo, parece não ser mais estratégica para os setores políticos que assumiram o poder de estado. tornou-se o quinto país a possuir os títulos do Tesouro Estadunidense - T-Bonds, ao duplicar em 210 bilhões de dólares o valor de compra desses títulos em menos de três anos, ou seja, em 2007, ele era o $17^{\circ}$ país detentor desses títulos, com 105 bilhões de dólares.

Com toda a crise política, o Brasil manteve até o último dia (31 de agosto de 2016) sob a presidência Dilma Rousseff em torno de 376,9 bilhões de dólares em títulos do Tesouro Estadunidense. Esse caixa em divisas internacionais era um fator de segurança que poderia ser acionado, caso houvesse o agravamento e extrema fragilização das contas públicas no cenário nacional.

40 Mesmo assim, ainda havia outros mecanismos que podiam deter o processo de deterioração das contas públicas: a repatriação do dinheiro de 8.667 contas de sonegadores depositadas no exterior poderia ser também um instrumento eficaz na redução desse processo.

41 No equilíbrio das forças econômicas e militares, considerando as zonas de fronteiras, as forças econômicas que representam o capitalismo periférico brasileiro, no caso as multinacionais brasileiras, possuíam um papel muito ativo. Como agentes da internacionalização da forma periférica de acumulação do capitalismo, essas empresas encontram-se no banco dos réus, passando por um julgamento totalmente improcedente por agir em favor do desenvolvimento da economia nacional, pois essas empresas contribuíram para a geração de empregos para o desenvolvimento econômico, e o modus operandi é bastante semelhante ao "lobismo" desenvolvido nos países capitalistas do Norte.

42 Com relação ainda às multinacionais brasileiras, o que estamos assistindo é o recrudescimento da zona de influência internacional dessas empresas, há deliberadamente forças ou interesses internacionais geopolíticos que estão atuando para reduzir o espaço de influência dessas empresas, o que vem acontecendo em todo o Mercosul, em que as forças neoliberais estão paralisando edificações, construções de estradas, portos, e empreendimentos de infraestrutura para expansão do Mercosul.

O elemento propulsor dessa ação é o prolongamento da crise nas economias centrais do Norte e a necessidade de expansão e de criação de novas frentes de serviços ou atividades, que possam representar a formação de novas fronteiras de acumulação econômica, como 
é o caso do pré-sal brasileiro, a mais importante reserva de petróleo no atlântico sul ou a maldição do ouro-negro brasileiro.

\section{A ascensão de setores favoráveis à globalização subordinada}

\section{Conclusão}

Quando os poderes públicos violam as liberdades fundamentais e os direitos garantidos pela Constituição, a resistência à opressão é um direito e um dever do cidadão. (AGANBEM, 2004, p. 23)

Os setores golpistas vem implementando, de forma açodada, programas de "austeridade" ultraneoliberais e reformas estruturais, através de Projetos de Emendas Constitucionais - 
PEC, que têm como meta flexibilizar/precarizar as relações trabalhistas, aumentar a jornada laboral, reformar a previdência social e destruir importantes conquistas sociais, embora todos os estudos apresentassem dados que comprovam que não há dificuldades orçamentárias na previdência, mas sonegação, elisão fiscal e desvio de recursos desse patrimônio dos trabalhadores brasileiros.

No capitalismo ultraneoliberal, o discurso que se hegemoniza apregoa o estado mínimo para os trabalhadores e a maioria do andar de baixo: austeridade, reforma trabalhista, reforma previdenciária, fim da saúde e da educação pública; para a minoria do andar de cima, empresários e representantes do capital, o estado máximo: isenções e anistia fiscal, eliminação de tributos, incentivos e investimentos financeiros.

Embora, a luta pela restauração da democracia e por eleições diretas, no Brasil, vem enfrentando uma dura resistência dos setores golpistas, cresce o sentimento de indignação, de resistência e de repúdio às políticas ultraneoliberais de um governo, que quer permanecer no poder até 2020, negligenciando a legitimidade do voto popular; cresce, também, a compreensão de que resistir à opressão e à destruição do estado social é um direito e um dever do cidadão.

\section{BIBLIOGRAFIA}

AGANBEM, G. Estado de Exceção. São Paulo: Boitempo, 2004.

HEIDEMANN, H. D.. O Estado Burguês: Um conceito básico da Geografia Política. Recife, UFPE: Revista de Geografia, I (1), set/dez, 1983.

JESSOP, B. A globalização e o Estado nacional. Revista Crítica Marxista: São Paulo, Xamã, v.1, n.7, 1998, p.9-45.

PIRES, H.F. Globalização e Integração Financeira e Tecnológica entre os Países Emergentes: 0 Novo Banco De Desenvolvimento do Brics. In: Revista Geo UERJ, Rio de Janeiro, № 27, pp.283-292, 2015. <http://www.e-publicacoes.uerj.br/index.php/geouerj/article/view/18952/14670>

Desregulação financeira no capitalismo gestor de dinheiro: O endividamento dos EUA e das economias centrais. Biblio 3W. Revista Bibliográfica de Geografía y Ciencias Sociales. Barcelona: Universidad de Barcelona, 25 de febrero de 2012, Vol. XVII, no 963. <http:// www.ub.es/geocrit/b3w-963.htm>

"Ethos" e mitos do pensamento único globaltotalitário. São Paulo: Revista Terra Livre (16), pp.153-167, 2001. <http://www.agb.org.br/publicacoes/index.php/terralivre/article/ download/354/336>

PORTER, M.E. A Vantagem Competitiva, das Nações. Rio de Janeiro: Campus, 1993.

SANTOS, M. Por uma outra globalização: do pensamento único à consciência universal.

Rio de Janeiro: Record, 2000. 


\section{NOTAS}

1. Artigo baseado na Conferência apresentada no Museu MUDEC/Milão, tendo como tema: “Globalização, cultura e território: o Brasil no novo milênio", em 10 de Maio de 2017, encontro organizado pela Iulm Università. Cf. In: <https://www.facebook.com/events/1410651658998120/> 2. Conferir em Pires, 2001.

3. Ver Porter, 1993.

4. Santos, 2000.

5. Conferir o projeto "Uma Ponte para o Futuro", em: http://pmdb.org.br/wp-content/ uploads/2015/10/RELEASE-TEMER_A4-28.10.15-Online.pdf

6. Mais informações sobre a PEC 241/55, conferir em: http://www.camara.gov.br/ proposicoesWeb/fichadetramitacao?idProposicao $=2088351$

7. Conferir no portal do Andes "Temer tira R\$ 4,3 bilhões do orçamento do Ministério da Educação”, em: http://portal.andes.org.br/andes/print-ultimas-noticias.andes?id=8734

8. Pires, 2015.

9. Consultar Pires, 2012.

\section{RESUMOS}

O título desse artigo foi sugerido pelo Professor Dr. Angelo Turco, em maio de 2017, para que eu pudesse apresentar um rápido painel sobre o contexto da globalização, da cultura e do Brasil, no período atual, durante minha estância como professor visitante na Faculdade de Artes, Turismo e Market, da Universidade Livre de Línguas e Comunicação de Milão, na Itália - IULM (Libera Università di Lingue e Comunicazione di Milano). Neste trabalho, analiso como o golpe jurídicopolítico midiático desfechado, em 31 de agosto de 2016, tornou hegemônico o discurso de setores pró-mercado/ultraneoliberais, que acreditam que a globalização é um imperativo irreversível do mercado e das empresas globais e que o estado deve adotar medidas de ajustamentos "subordinados" aos imperativos da globalização. Entretanto, como Milton Santos, defendo a premissa de que é preciso resistir e desenvolver uma globalização solidária, não subordinada, que promova um conjunto de políticas públicas alternativas que possibilitem a formação de uma sociedade com menos desigualdades na distribuição de renda e que também promovam a elevação no padrão de vida dos grupos sociais prejudicados com as reformas atuais das relações de trabalho e perda dos benefícios previdenciários assegurados pela legislação.

The title of this article was suggested by Professor Dr. Angelo Turco, in May 2017, so that I could present a quick panel on the context of globalization, culture and Brazil, in the current period, during my stay as visiting professor at the Faculty of Arts, Tourism and Market, of the Free University of Languages and Communication of Milan, in Italy - IULM (Libera Università di Lingue and Comunicazione di Milano). This paper investigates how the media-political coup occurred on August 31, 2016, amplified the agenda from pro-market and ultraneoliberal sectors that assume globalization is an irreversible imperative of the market as well as global companies and what is more, that the state should adopt adjustment measures subdued to globalization imperatives. As Milton Santos once did, we defend that it is necessary to resist and develop some 
kind of solidary globalization - not subdued - that promotes alternative public policies to enable the constitution of a society that suffers less from inequality concerning income distribution and can also advance in standard of living of social groups most affected by current reforms of labor relations and loss of pension benefits.

El título de este artículo fue sugerido por el Profesor Dr. Angelo Turco, en mayo de 2017, para poder presentar un rápido panel sobre el contexto de la globalización, la cultura y Brasil, en el período actual, durante mi estancia como profesor visitante en la Facultad de Artes, Turismo y Marketing, de la Universidad Libre de Lenguas y Comunicación de Milán, en Italia - IULM (Libera Università di Lingue y Comunicazione di Milano). En este trabajo, se analiza como el golpe jurídico-político mediático desencadenado, el 31 de agosto de 2016, hizo hegemónico el discurso de sectores pro-mercado / ultra-neoliberales, así como de empresas globales, que creen que la globalización es un imperativo irreversible del mercado y que el Estado debe adoptar medidas de ajustes "subordinados" a los imperativos de la globalización. Sin embargo, como Milton Santos, defiendo la premisa de que hay que resistir y desarrollar una globalización solidaria, no subordinada, que promueva un conjunto de políticas públicas alternativas que posibiliten la formación de una sociedad con menos desigualdades en la distribución de la renta y que también promuevan un mejoramiento de la calidad de vida de los grupos sociales perjudicados con las reformas actuales de las relaciones de trabajo y pérdida de la seguridad social establecida por la legislación.

Le titre de cet article a été proposé par le professeur Angelo Turco en mai 2017, pour que je puisse présenter un brief panel sur le contexte de la mondialisation, de la culture et du Brésil, dans l'actualité, pendant mon séjour en tant que professeur invité à la Faculté Arts, Tourisme et Marketing de l'Université libre de langues et de la communication de Milan, Italie - IULM (Libera Università di Lingue e Comunicazione di Milano). Dans cet article, j'analyse comme le coup juridique-politique des médias, qui a eu lieu le 31 Août 2016, a rendu hégémonique le discours de secteurs pro-marché / ultranéolibéraux qui croient que la mondialisation est un impératif irréversible du marché et des entreprises mondiales et qui l'Etat doit adopter des mesures d'ajustements «subordonnés» aux impératifs de la mondialisation. Cependant, comme Milton Santos, je soutiens la prémisse qu'il faut résister et développer une mondialisation solidaire, non soumise, qui favorise un ensemble de politiques publiques alternatives qui permettent la formation d'une société avec moins d'inégalités dans la répartition des revenus et qui favorisent également la augmentation du niveau de vie des groupes sociaux entravée par les réformes actuelles des relations de travail et la perte des prestations de sécurité sociale défavorisés par les reformes actuelles des relations de travail et par la perte des bénéfices de sociaux assurés par la législation.

\section{ÍNDICE}

Palavras-chave: Globalização, Cultura, Brasil, Golpe Jurídico-Político Midiático.

Palabras claves: Globalización, Cultura, Brasil, Golpe Jurídico-Político Mediático.

Keywords: Globalization; Culture; Brazil; Media-political Coup.

Mots-clés: Mondialisation, Culture, Brésil, Coup juridique- politique des media. 
AUTOR

HINDENBURGO FRANCISCO PIRES

Universidade do Estado do Rio de Janeiro. Instituto de Geografia

e-mail: hindenburgo@uerj.br 